ORIGINAL ARTICLE

\title{
Different Endoscopic Approaches Addressing Frontal Sinus
}

\section{Mohamed Kamal Mobashir, Nasser Naguib Nasr, Ahmed Mohamed Elhady Anany, Ahmed Emad El Din El Maghawry El Sayed Askar}

\section{Otorhinolaryngology Department, Faculty of Medicine, Zagazig University, Zagazig, Egypt}

Corresponding author :

Ahmed Emad El Din El

Maghawry El Sayed

Askar

Otorhinolaryngology

Department, Faculty of

Medicine, Zagazig

University, Zagazig,

Egypt

E-mail:

a.emad234@gmail.com
Background: Chronic frontal sinusitis as a component of chronic rhinosinusitisis is one of the greatest challenges in rhinology. The endoscopic endonasal approach to the frontal sinus became the standard approach in chronic rhino sinusitis refractory to medical management.

Objectives: The aim of this work is to assess different approaches addressing frontal sinus disease in twenty four patients with chronic frontal sinusitis resistant to medical treatment for a period not less than twelve weeks in Otorhinolaryngology Department in Zagazig University Hospitals from March 2018 to November 2018.

Patients and methods: Patients' age ranged from 20 to 58 years with a mean of $32.6 \pm 10.68$. These patients had two different approaches on each nasal side: Zero angled endoscopic approach (to perform standard uncinectomy and anterior ethmoidectomy including resection of ethmoidal bulla with middle turbinate preservation then removal of agger nasi cells accessing frontal sinus) and resection of the superior attachment of uncinate process (with preservation of ethmoidal bulla then using 70 degree angled endoscope to expose the frontal sinus).

Results: In both techniques, preservation of ethmoid artery, middle turbinate and lamina papracyae were maintained. Residual mucopurulent discharge in the frontal recess area was found in 4 sides (10\%) and managed medically. Endoscopic assessment revealed lateralization of middle turbinate in 6 sides only (15\%) which had ethmoidectomy prior to frontal sinusotomy.

Conclusion: Addressing frontal sinus through intact bulla technique is less invasive guarding against anterior ethmoidal artery injury, scarring at frontal recess area and frontal ostium restenosis.

Key Words: frontal sinus, endoscopy, intact bulla.

\section{INTRODUCTION}

T he frontal sinus shares a common embryological and anatomical relationship with the ethmoid sinus, to the point that several authors and researchers have referred to this sinus as a "large ethmoidal cell" or simply the termination or upper limit of the intricate ethmoidal labyrinth. Two frontal sinuses are usually seen. Each frontal sinus cavity takes on the shape of a pyramid, with a thick anterior table and a thinner posterior table ${ }^{(\mathbf{1})}$.
Chronic rhinosinusitis is characterized by two or more symptoms, one of which should be either nasal blockage ,obstruction, congestion or nasal discharge (anterior or posterior nasal drip) and the other may be facial pain or reduction or loss of smell for equal to or more than 12 weeks. This should be supported by demonstrable disease either endoscopic signs of nasal polyps or mucopurulent discharge from middle meatus and CT changes showing mucosal changes within the osteomeatal complex or sinuses ${ }^{(2)}$. 
The frontal sinus outflow tract (FSOT) can be envisioned as an hourglass with three basic components; frontal sinus infundibulum, frontal sinus ostium and frontal recess. Failure to maintain the frontal sinus outflow tract patent (because of edema, fibrosis, polyps and/or neoplasm) may trigger a vicious cycle of events that results in retained secretions, secondary bacterial colonization, hypoxia, $\mathrm{pH}$ changes and ciliary dysfunction. Any or all of these physiological changes may culminate in chronic rhinosinusitis ${ }^{(3)}$.

Patients with chronic frontal rhinosinusitis frequently have associated disease in the remaining paranasal sinuses. Diagnostic nasal endoscopy is the most comprehensive physical examination for the rhinologic patient. In patients who have undergone previous surgery, frontal sinus disease is suspected when the following are seen: Lateralized or amputated middle turbinate, synechia and polypoid edema in the anterior ethmoid cavity ${ }^{(4)}$.

There is no medical therapy designed specifically for the frontal sinus. The choice of therapeutic agents should be made thoughtfully and on an individualized basis. Adjuvant therapy focusing on the reduction of inflammation is also frequently recommended. Adjuvant therapy in chronic frontal sinusitis may include intranasal and systemic steroids, topical and systemic decongestants, antihistamines, leukotriene modifiers, mucolytics and nasal saline nasal spray $^{(5)}$.

The first frontal sinus procedure was described in $1750^{(6)}$. The recent advances in imaging and endoscopic techniques have resulted in the resurgence of intranasal procedures for the treatment of frontal sinus disease. Frontal sinus disease, particularly chronic frontal sinusitis, is a highly morbid and sometimes life-threatening condition because of its potential complications. Despite the fact that over the years the incidence of complications has decreased, orbital and intracranial complications, including meningitis, subdural abscess, intracerebral abscess, and osteomyelitis continue to occur ${ }^{(4)}$.

The aim of this study is to assess different approaches addressing frontal sinus disease.

\section{PATIENTS AND METHODS}

\section{Patients:}

This study included twenty four patients with chronic frontal sinusitis resistant to medical treatment for a period not less than twelve weeks. The study work was done in Otorhinolaryngology Department in Zagazig University Hospitals for duration from March 2018 to November 2018. Patient age ranged from 20 to 58 years with a mean $\pm \mathrm{SD}$ of $33.54 \pm 12$. They are 6 males and 18 females. Written informed consent was obtained from all participants and the study was approved by the research ethical committee of Faculty of Medicine, Zagazig University. The work has been carried out in accordance with The Code of Ethics of the World Medical Association (Declaration of Helsinki) for studies involving humans.

Table (1) showing inclusion and exclusion criteria.

\section{Methods:}

These patients were subjected to the following:

1- History taking.

2- General examination.

3- Nasal examination (anterior and posterior rhinoscopy and nasal decongestion).

4- Nasal endoscopy reporting on presence of polyp, discharge and edema and exclusion of other nasal pathologies.

5- Radiological evaluation in the form of computed tomographic (CT) scanning with Phillips ICT BRILLIANCE 256 slice, $1 \mathrm{~mm}$ cuts (Figure 1).

6- $\quad$ Presurgery treatment consisted of a topical steroid for all cases with chronic sinusitis or chronic sinusitis with nasal polyposis and 
short intermittent doses of oral steroids only for those cases of chronic sinusitis with nasal polyposis, antihistaminic and antibiotic if there is infection.

7- Patients had two different approaches on each nasal side:

- Intact bulla approach: Starting with Zero angled endoscope, we elevate uncinate process by ball probe then using a back biting forceps to remove the middle part of uncinate process and localization of maxillary ostium. Next we remove the horizontal part of the uncinate process then elevation of the vertical part of the uncinate process until its upper attachment and its removal. We now shift to 70 degree angled endoscope and localize agger nasi air cells. Removal of anterior, medial and posterior wall of agger nasi is done then removal of frontal air cells. We now localize the boundaries of frontal sinus ostium where medially we have the middle turbinate, anteriorly the frontal peak, laterally lamina papracyae and posteriorly the scalloping of the frontal sinus. If there is a supra orbital recess, removal of the bony partition between it and the frontal sinus (8) (Figure 2).

- Classic approach: Standard uncinectomy and anterior ethmoidectomy is first performed with middle turbinate preservation. The superior attachment of the uncinate process is removed under visualization and using thrucutting frontal sinus punch forceps. At this time, the variable bony partitions within the frontal recess are identified and the anatomic relationships corresponded to the preoperative imaging. In most cases, the uncinate will attach laterally on the upper orbital wall, creating a "recessus terminalis" that may be continuous with a pneumatized agger nasi cell system anteriorly. Removal of the remaining uncinate process insertion and "uncapping" of the agger nasi cell will frequently expose the internal frontal sinus ostium. ${ }^{(7)}$

\section{Postoperative care:}

After the operation, those patients who underwent FESS were taken out from the operating room and into a recovery room for observation.

- Pack removal after 48 hour.

- Parenteral broad spectrum antibiotics for one week.

- Analgesics.

- Nasal decongestants three times daily for 5 days.

- Close observation for postnasal bleeding or other complications.

- Alkaline nasal douches for at least three weeks.

- Intranasal steroids in regular courses.

- Medical treatment and evaluation of the general condition.

\section{Follow up after surgery:}

Follow up weekly for first month, then, monthly for first 3 months (Figure 3).

\section{STATISTICAL ANALYSIS}

Data were entered checked and analyzed using Epi-Info version 6 and SPP for Windows version 8. Data were summarized using the arithmetic mean, standard deviation, median, student $t$ test and chi-squared test. ${ }^{\left({ }^{(6)}\right.}$

\section{RESULTS}

This study included 24 patients. 6 patients $(25 \%)$ were males and 18 patients $(75 \%)$ were females. Their age ranged from 20 to 58 with mean age $33.54 \pm 12$ years old.

All patients complained from headache/facial pain, $79.2 \%$ of hyposmia and nasal obstruction, $70.8 \%$ of post nasal secretions, $20.8 \%$ of rhinorrhea and $12.5 \%$ of cough (Table 2).

The studied patients had irrelevant past history, history of asthma or trauma.

In our study, 10 patients $(41.7 \%)$ had bilateral ethmoido-frontal polyposis, 4 (16.7\%) had bilateral panpolyposis, 4 (16.7\%) had chronic pansinusitis, $3(12.5 \%)$ had isolated frontal sinusitis and $3(12.5 \%)$ had allergic fungal sinusitis. 17 patients $(70.8 \%)$ 
had septal deviations, 11 of them (45.9\%) needed endoscopic correction before FESS (Table 3).

According to the Lund-MacKay CT staging, the mean score for the frontal sinus was $2.83(1=$ partial opacification, $2=$ complete opacification).

The largest percentage of the studied patients had bilateral lesion $(66.7 \%)$. Those 16 patients underwent both classic approach (for right lesions) and intact bullae technique (for left side lesions). 3 of patients having right unilateral lesions underwent classic approach while other five lesions were on left side and underwent intact bullae technique. So we did 19 side through classic approach and 21 side through intact bullae technique.

In both techniques, preservation of ethmoid artery, middle turbinate and lamina papracyae were maintained.

There is highly statistically significant difference between the two surgical techniques regarding operative duration. Intact bullae technique had the least duration with a mean of 26.14 minutes compared to Classic approach with a mean of 35.47 minutes.

As regards postoperative complications, no major complications (significant hemorrhages, orbital complications or cerebrospinal fluid leak) occurred. At the end of follow-up period ( 3 months), all patients were assessed subjectively by presence of postoperative symptoms and objectively by postoperative nasal endoscopic examination and CT scan.

Subjective assessment revealed no recurrence of symptoms (frontal discomfort, headache or pain). Objective postoperative endoscopic assessment revealed nasal adhesions between inferior turbinate and septum in 4 sides only $(10 \%)$ that were excised locally at outpatient clinic.

Endoscopic assessment revealed lateralization of middle turbinate in 6 sides only $(15 \%)$ which had ethmoidectomy prior to frontal sinusotomy with no symptoms or signs of disease recurrence. There was no middle turbinate lateralization in cases of isolated frontal sinusitis whose ethmoidal bulla remained intact denoting that preserving the ethmoidal bulla guards against middle turbinate lateralization.

Residual mucopurulent discharge in the frontal recess area with endoscopic mucosal edema were found in 4 sides $(10 \%)$ and were managed medically. No patients required revision sinus surgery and all complications were managed medically (Table 4).

No patient who underwent classic or intact bullae technique developed recurrence or frontal ostium restenosis by the end of the 3 months follow up period. The less invasiveness of the intact bulla technique will guard against any frontal ostium stenosis on the long run 
Table (1): Inclusion and exclusion criteria

\begin{tabular}{|c|c|}
\hline Incl & Exclusion criteria \\
\hline $\begin{array}{l}\text { - All patients proved to have sinusitis for more } \\
\text { than three months duration not responding to } \\
\text { medical } \\
\text { treatment } \\
\text { - Patient must be fit for surgery. }\end{array}$ & $\begin{array}{l}\text { - Patients with chronic sinusitis who responded to } \\
\text { medical treatment. } \\
\text { - Patients who were not willing to undergo ESS. } \\
\text { - Patients with osteomyelitis and infiltrating tumors. } \\
\text { - Revision cases with previously preformed anterior } \\
\text { ethmoidectomy. }\end{array}$ \\
\hline
\end{tabular}

Table (2): Presenting symptoms in the studied patients

\begin{tabular}{|l|c|c|}
\hline & $\mathbf{N}$ & \% \\
\hline Headache/facial pain: & 0 & 0 \\
Absent & 24 & 100 \\
Present & 5 & 20.8 \\
\hline Nasal obstruction & 19 & 79.2 \\
Absent & 19 & 79.2 \\
Present & 5 & 20.8 \\
\hline Rhinorrhea: & 5 & 20.8 \\
Absent & 19 & 79.2 \\
Present & & \\
Hyposmia: & 7 & 29.2 \\
Absent & 17 & 70.8 \\
Present & 21 & \\
\hline Postnasal secretion: & 3 & 12.5 \\
Absent & & \\
Present & & \\
\hline Cough: & & \\
Absent & & \\
Present & & \\
\hline
\end{tabular}

Table (3): Endoscopic features of the studied patients

\begin{tabular}{|c|c|c|}
\hline & $\mathbf{N}$ & $\%$ \\
\hline $\begin{array}{l}\text { Endoscopic features: } \\
\text { Bilateral ethmoido-fronta1 polyposis } \\
\text { Left isolated frontal sinusitis } \\
\text { chronic pansinusitis } \\
\text { Panpolyposis } \\
\text { Right allergic fungal sinusitis }\end{array}$ & $\begin{array}{l}10 \\
3 \\
4 \\
4 \\
3\end{array}$ & $\begin{array}{l}41.7 \\
12.5 \\
16.6 \\
16.7 \\
12.5\end{array}$ \\
\hline $\begin{array}{l}\text { Septum: } \\
\text { NAD } \\
\text { Deviated septum to the left } \\
\text { - Corrected endoscoipcally } \\
\text { - Need no intervention } \\
\text { Deviated septum to the right: } \\
\text { - Corrected endoscoipcally } \\
\text { - Mid high septal deviation need no intervention }\end{array}$ & $\begin{array}{l}7 \\
4\end{array}$ & $\begin{array}{c}16.7 \\
8.3\end{array}$ \\
\hline
\end{tabular}


Table (4): Postoperative complications in the studied patients

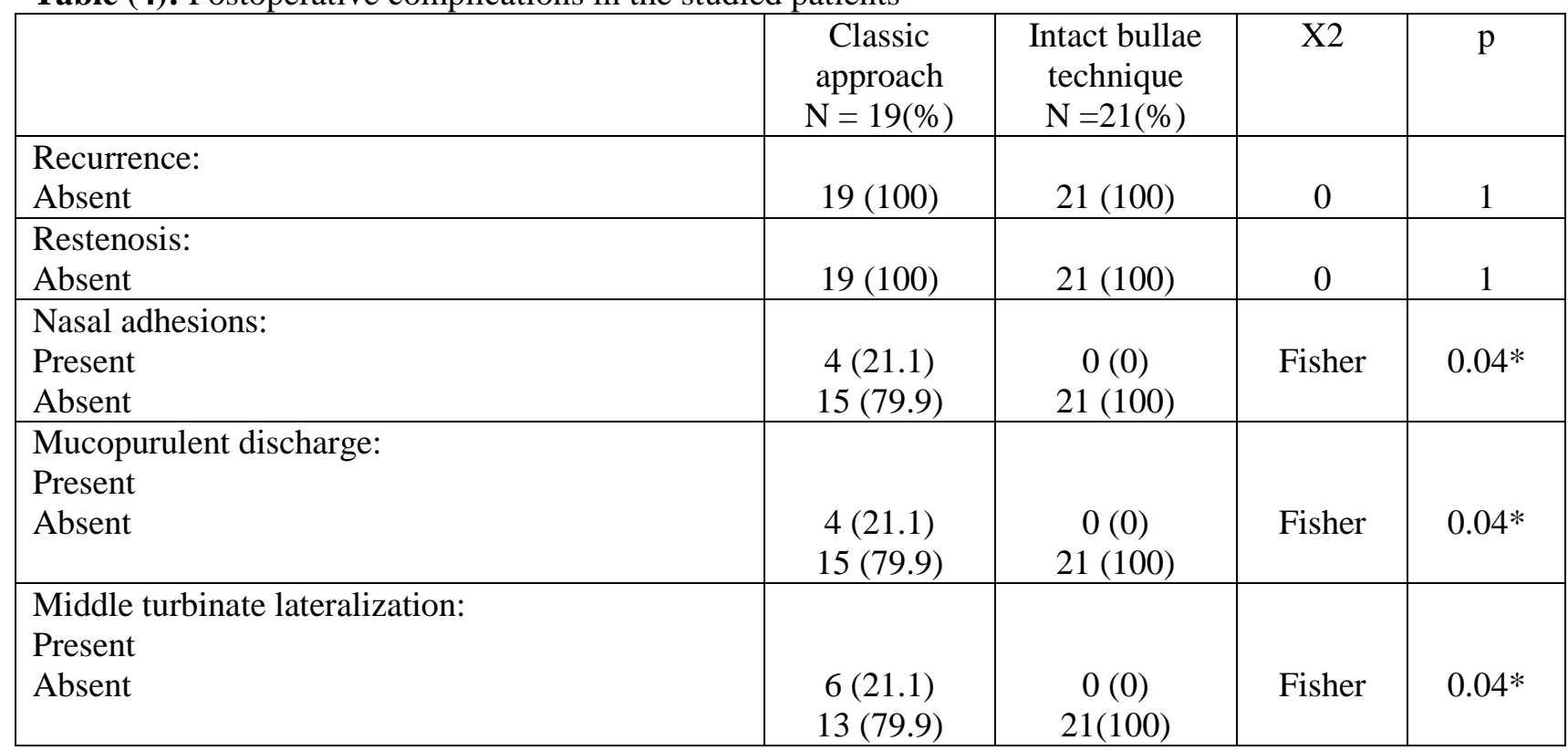

$*_{\mathrm{p}}<0.05$ is statistically significant

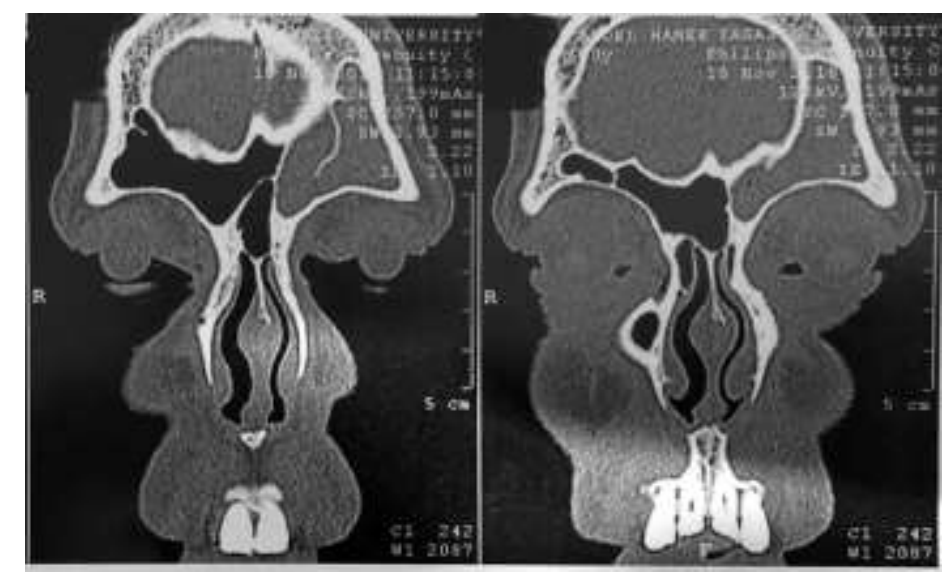

Fig.1 CT coronal cuts of a case of Left isolated frontal sinusitis. 


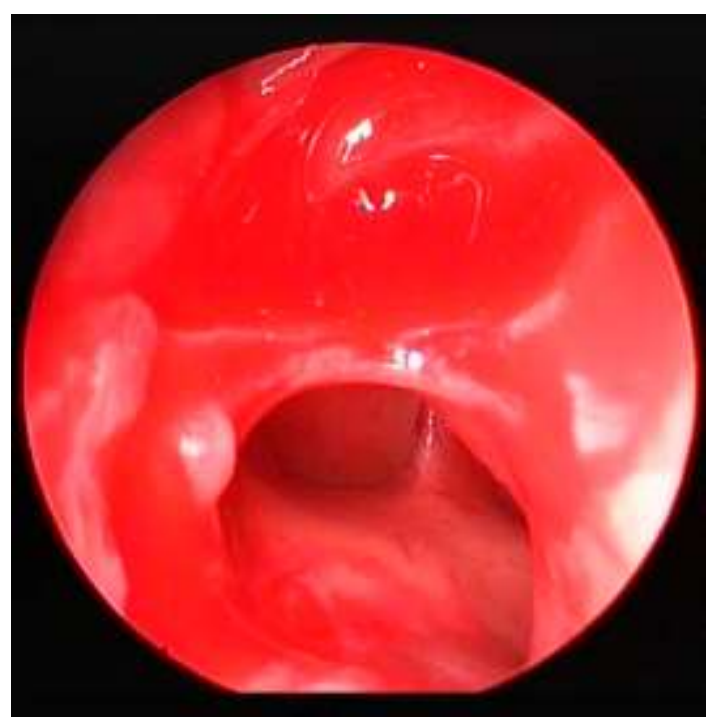

Fig.2 70 degree endoscopic view of frontal sinus ostium through intact bulla technique.

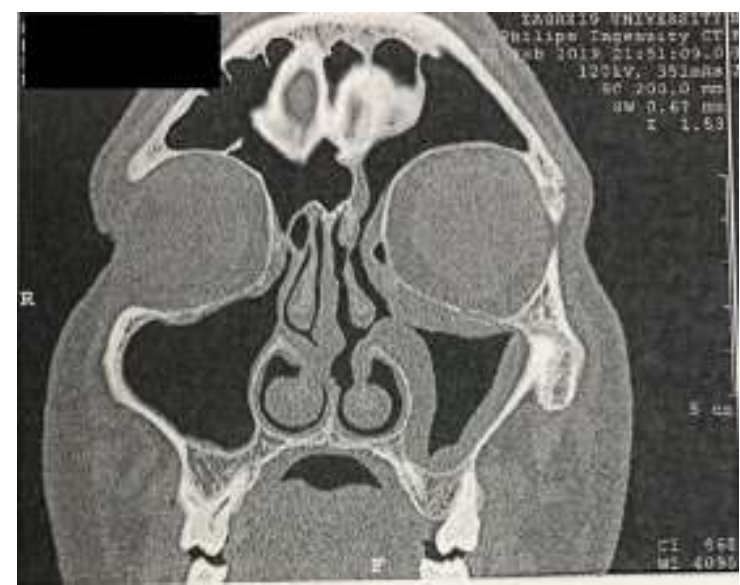

Fig.3 CT coronal cut 3 months post operative of the same case of Left isolated frontal sinusitis

\section{DISCUSSION}

Frontal sinusitis is a common illness and usually secondary to inflammatory changes in the pre-chambers, i.e., the anterior ethmoid cells; hence the rationale for treating chronic frontal sinusitis by endoscopic anterior ethmoidectomy. In contrast, isolated frontal sinusitis is infrequently encountered. In this condition, most of the ethmoid cells are well aerated and the frontal sinus is involved secondary to anatomical obstruction or inflammatory changes confined to the frontal recess. ${ }^{(7)}$

Circumscribed forms of chronic sinusitis usually originate from micro anatomic anomalies, especially narrow passages or clefts with areas of pathological mucosal contact. These occur mainly in the middle nasal meatus, in the region of the ostiomeatal unit. They impair mucociliary clearance and ventilation of the functionally dependent paranasal sinuses. Most of the cases demonstrating frontal or maxillary sinus opacification have anterior ethmoid opacification as well. ${ }^{(8)}$

The functional term "ostiomeatal unit" refers to the anatomic region lateral to the anterior two-thirds of the middle turbinate. It includes the uncinate process, the hiatus semilunaris, the maxillary ostium, the ethmoid bulla, the remaining anterior ethmoid cells, and the frontal recess. Because the frontal sinus is subordinate to this complex, routine surgical management of chronic 
frontal sinusitis consists of anterior ethmoidectomy that includes removal of the ethmoid bulla and the remaining anterior ethmoid cells. ${ }^{(8)}$

The rationale in this study is to compare between intact bulla ethmoidalis technique in addressing the frontal sinus and the classic technique of anterior ethmoidectomy prior to frontal sinusotomy regarding the invasiveness of the operation and the time elapsed till identification of the frontal sinus ostium.

In a computer-assisted anatomical study of the frontal recess, Landsberg and Friedman (9) investigated variations in the superior attachment of the uncinate process, the prevalence of agger nasi, and the dimensions of the frontal ostium. A simplified endoscopic surgical approach to the frontal sinus was designed by applying this knowledge. This approach emphasizes complete removal of the superior attachment of the uncinate process, including the terminal recess, if frontal sinusotomy is indicated. They concluded that chronic isolated frontal sinusitis can be effectively treated in selected cases with a minimally invasive endoscopic procedure that is limited to the reestablishment of frontal sinus outflow. Landsberg et al. ${ }^{(8)}$ failed to reveal either a patent frontal sinus or transillumination by endoscopy.

Setliff et al. ${ }^{(10)}$ found that $68 \%$ of the ethmoid bullae have a single posterior opening into the hiatus semilunaris superior. This may explain the occasional presence of frontal recess and sinus disease without spread of infection into the ethmoid bulla and the other anterior ethmoid cells. Hence, in certain cases, the frontal sinus is subordinate only to the most anterior compartment of the ostiomeatal unit, namely, the frontal recess.

The concept of "intact bulla sinusotomy" was introduced by Louri (11). Landesberg et al. ${ }^{(8)}$ developed and refined the technique and were encouraged by the frequent absence of communication between the frontal recess and the ethmoidal bulla. The combined circumstances inevitably paved the way to treatment of chronic isolated frontal sinusitis by sinusotomy alone.

In several anatomic variations, such as frontal recess pneumatization originating behind the plane of the bulla lamella, the described technique may be insufficient. Supraorbital ethmoid cells, suprabullar cells, and frontal bullar cells are not accessible if the ethmoid bulla is not dissected. Hence, the ethmoid bulla should be resected if these cells appear preoperatively or intraoperatively to be involved in the inflammatory process. Thus, the "intact bulla" technique is applicable in selected cases, when these anatomical variants are not present. ${ }^{(12)}$

The number of skilled surgeons practicing ESS is rapidly growing and the surgical technique becomes more sophisticated as more experience is gained. Each case should be individually planned and, where possible, ESS should be minimally invasive. Standardized surgery for different disease patterns should be avoided. Just as maxillary sinusitis can be treated without ethmoidectomy and sphenoid sinusitis without ethmoidectomy, isolated frontal sinusitis can be treated without complete anterior ethmoidectomy. ${ }^{(13)}$

In our study according to the LundMacKay CT staging, the mean score for the frontal sinus opacification was $2.83 \pm 0.7$ compared to Landsberg et al. (8) who mentioned that the mean score for the frontal sinus was $1.6(1=$ partial opacification, $2=$ complete opacification).

Various approaches to the frontal recess have been recommended. Stammberger et al. (7) advocated clearance of the frontal recess from below, using angled telescopes and curved instruments. This technique becomes increasingly difficult if frontal cells extend toward the skull base, especially if they reach the skull base and impinge on the frontal recess or frontal sinus. It is rare, and happens only when access is very broad, that a direct 
view into the frontal sinus can be obtained. However, the surgical principle that the better the access the easier the surgical procedure is valid for endoscopic sinus surgery.

Difficulty is also increased if significant polyposis and mucosal disease are present because these increase the vascularity of the tissues. The technical skill required to work with angled telescopes and angled instruments is also considerable. ${ }^{(\mathbf{1 4})}$

In our study, 17 patients $(70.8 \%)$ had septal deviations, 11 of them $(45.9 \%)$ needed prior endoscopic correction to allow access to frontal recess.

Landsberg et al. ${ }^{(8)}$ left the ethmoid bulla intact in all 11 patients and served as the posterior boundary of dissection. The middle turbinate was preserved in all but 1 patient who underwent bilateral middle turbinoplasty. Three patients underwent a limited septatoplasty in which a piece of cartilage or bone was resected at the level of the middle turbinate in order to facilitate surgical access to the middle meatus.

In our study, Objective postoperative endoscopic assessment of all patients revealed no recurrence of original disease or frontal ostium restenosis. The less invasiveness of intact bulla technique decreases incidence of scarring at the frontal recess area and guards against frontal sinus ostium restenosis on the long run.

Sethi and Marple ${ }^{(15)}$ have shown that one of the commonest causes of the failure of endoscopic surgery of the sinuses is persistence of original disease in the frontal recess due to incomplete surgery. Injudicious dissection of the frontal recess, excessive mucosal trauma resulting in an unstable middle turbinate which lateralizes to obstruct the frontal outflow tract is common cause of iatrogenic disease of the frontal sinus.

In our study, no major complications (significant hemorrhages, orbital complications or cerebrospinal fluid leak) occurred. Objective postoperative endoscopic assessment revealed nasal adhesions between inferior turbinate and septum in 4 sides only $(10 \%)$ that were excised locally at outpatient clinic.

Residual mucopurulent discharge in the frontal recess area with endoscopic mucosal edema were found in 4 sides $(10 \%)$ and were managed medically. No patients required revision sinus surgery and all complications were managed medically.

Landsberg et al. (8) found no major complications. Two patients had small synechiae between the middle turbinate and the lateral nasal wall. They concluded that chronic isolated frontal sinusitis can be effectively treated in selected cases by a targeted endoscopic procedure, limited to reestablishment of frontal sinus outflow.

Complications of frontal recess surgery result from an inadequate understanding of the anatomy, difficulty in identifying the frontal recess intraoperatively, difficulty in working with angled endoscopes and instruments while attempting to preserve mucosa in an area which is bounded by the skull base and the orbit. ${ }^{(15)}$

In our study, endoscopic assessment revealed lateralization of middle turbinate in 6 sides only $(15 \%)$ which had ethmoidectomy prior to frontal sinusotomy with no symptoms or signs of disease recurrence. There was no middle turbinate lateralization in cases of isolated frontal sinusitis whose ethmoidal bulla remained intact denoting that preserving the ethmoidal bulla guards against middle turbinate lateralization.

Middle turbinate lateralization (MTL) is one of the most common complications of endoscopic sinus surgery, with incidence reported as high as $43 \%$ and, therefore, various techniques have been described in an attempt to decrease lateralization. MTL potentially decreases drainage of frontal, anterior ethmoid and maxillary sinuses in addition to compromising the ability to 
debride the sinonasal cavity, irrigate and administer topical medications. ${ }^{(16)}$

MTL is among the most common explanations for endoscopic sinus surgery failure and need for revision surgery. During endoscopic sinus surgery, the middle turbinate is medialized to access the maxillary, frontal and ethmoid sinuses. The middle turbinate is thought to be further destabilized when the basal lamella is penetrated and when frontal recess clearance is performed. Subsequent epithelial damage to the lateral middle turbinate can occur as instruments are passed during surgery. Opposing raw mucosal surfaces predispose to synechia formation resulting in a turbinate held in a lateralized and obstructing position. The rate MTL after axillary flap approach to the frontal recess was approximately $15 \% .^{(16)}$

In our study, anterior ethmoidal artery, lamina papracyae and middle turbinate were preserved in all patients.

Although complete middle turbinate resection may appear reasonable to prevent lateral wall scarring, this maneuver remains highly controversial. A study by Henriquez et al. (16) suggested that partial middle turbinate resection had no significant benefit in reducing postoperative synechiae. Further, the middle turbinate is also an important surgical landmark and a higher risk of complications is observed in the absence of the middle turbinate during revision surgery.

There is highly statistically significant difference between the two surgical techniques regarding operative duration. Intact bullae technique had the least duration with a mean of 26.14 \pm 1.62 minutes compared to Classic approach with a mean of $35.47 \pm 2.65$ minutes and to our extent of knowledge, there is no published study discussing same issue.

The outcomes of chronic rhinosinusitis does not depend only on the approach but also depends on the nature of the disease (chronic rhinosinusitis with and without polyposis, allergic fungal sinusitis) and the extent of the disease whether it is isolated within the frontal sinus or associated with other diseases. So we recommend in further studies to address one type of chronic rhinosinusitis to avoid the confounding factors.

In our study, we faced a limitation that the operative duration till identification of frontal sinus ostium is affected by other factors such as nature of disease, intraoperative bleeding, and number of frontal recess cells that should be removed first. These factors should be excluded in any upcoming study.

One more limitation is that the follow up period is too short (only 3 months), we recommend that it should be minimally 6 months in any upcoming study.

\section{CONCLUSION}

Chronic frontal sinusitis develops secondary to frontal recess inflammatory changes and, in selected cases, can be surgically treated by a targeted endoscopic procedure limited to the reestablishment of frontal sinus outflow.

We concluded that addressing frontal sinus through intact bulla technique is less invasive and guards against anterior ethmoidal artery accidental injury during surgery. Also it is much easier and faster to identify the frontal sinus ostium during FESS operation through this approach saving time and effort for the surgeon.

\section{Declaration of interest}

The authors report no conflicts of interest. The authors alone are responsible for the content and writing of the paper.

\section{Funding information}

None declared

\section{REFERENCES}

1- Sadler TW. Langman's Medical Embryology, Ninth Ed. Lippincott Williams \& Wilkins, Philadelphia 2004; 363-401.

2- Ferguson BJ, Narita M, Yu VL, Wagener MM, Gwaltney JM. Prospective observational study of chronic rhinosinusitis: environmental 
triggers and antibiotic implications. Clin Infect Dis 2012;54:62-68.

3- Zeifer B. Update on sinonasal imaging anatomy and inflammatory disease. Neuroimaging Clin North Am 1998; 8: 607614.

4- Kountakis SE, Senior BA, Draf W. The Frontal Sinus. Springer-Verlag Berlin Heidelberg 2005; 1: 1-5.

5- Schlosser RJ, London SD, Gwaltney JM Jr, Gross CW. Microbiology of chronic frontal sinusitis. Laryngoscope 2001; 111(8): 133032.

6- Dean F. Statistical methods in scientific researches. European Journal of Scientific Research 2006; 14(3).

7- Stammberger H, Kopp W, Dekornfeld TJ, Hawke M. Functional endoscopic sinus surgery: the Messerklinger technique. In: Special Endoscopic Anatomy. Philadelphia: BC Decker 1991; 61-90.

8- Landsberg R, Segev Y, Friedman M, Fliss DM and Derowe A. A targeted endoscopic approach to chronic isolated frontal sinusitis. Otolaryngology; Head and Neck Surgery 2006; 134: 28-32.

9- Landsberg R, Friedman M. A computerassisted anatomical study of the frontal recess. Laryngoscope 2001; 111: 2125-30.

10- $\quad$ Setliff RC, Catalano PJ, Catalano LA. An anatomic classification of the ethmoid bulla.
Otolaryngol Head Neck Surg 2001; 125: 598602.

11- Louri MC. Endoscopic frontal recess and frontal sinus ostium dissection. Laryngoscope 1993; 103: 455-8.

12- Lee WT, Kuhn FA, Citardi MJ. 3D CT analysis of frontal recess anatomy in patients without frontal sinusitis. Otolaryngol Head Neck Surg 2004; 131: $164-73$.

13- Catalano P, Roffman E. Outcome in patients with chronic sinusitis after the minimally invasive sinus technique. Am J Rhinol 2003; 17: 17-22.

14- Kennedy DW, Senior BA. Endoscopic sinus surgery: a review. Otolaryngol Clin North Am 1997; 30: 313-330.

15- Sethi D and Marple B. Endoscopic Frontal Recess Surgery. American Academy of Otolaryngology-Head and Neck Surgery Foundation 2012; 1520-2.

16- Worldmald $P$. The agger nasi cell: The key to understanding the anatomyof the frontal recess. Otolaryngol Head Neck Surg 2003; 129(5): 497-507.

17- Henriquez OA, Schlosser RJ, Mace JC, Smith TL, Soler ZM. Impact of synechiae after endoscopic sinus surgery on long-term outcomes in chronic rhinosinusitis. Laryngoscope, 2013;123:2615-2619. 